\title{
Kapalı idealler üzerinde asal gamma halkanın kesirler halkası
}

\author{
Ahmet GENÇ* \\ Adnan Menderes Üniversitesi, Fen Edebiyat Fakültesi, Matematik. Bölümü, Merkez Kampüsü, Aydın \\ Gelis Tarihi (Received Date): 12.01.2021 \\ Kabul Tarihi (Accepted Date): 08.02.2021
}

$\ddot{\mathbf{O} z}$

Bu çalışmanın amacı, soldan kapalı idealler üzerinde Nobusawa anlamında asal gamma halkanın kesirler halkasını oluşturmaktır.

Anahtar kelimeler: Asal gamma halka, sol ideal, soldan kapalı ideal.

\section{Quotient rings of prime gamma ring on the closed ideals}

\begin{abstract}
The aim of this paper is to give a construction of quotient gamma rings of prime gamma rings in the sense of Nobusawa on left closed ideals.
\end{abstract}

Keywords: Prime gamma ring, left ideal, left closed ideal.

\section{Giriş}

Gamma halkalar teorisi hakkındaki çalışmalar Nobusawa' nın [7] 1964 yılında yaptığı çalışma ile başlamıştır. Bu çalışmasında Nobusawa, Wedderburn-Artin Teoreminin genelleştirilmesini ispatlamıştır. Günümüze kadar birçok yazar, gamma halkalar hakında çeşitli çalışmalar yapmışlardır. Birleşmeli halkalar (cebirler) teorisinde, (yarı) asal halkalar büyük bir sınıfı oluşturmaktadır. $\mathrm{Bu}$ teorideki sıklıkla çalışaılan bölüm genelleştirilmiş polinom özdeşliklerini (GPI) sağlayan (yarı) asal halkalardır. W. Martindale [6], aşikar olmayan genelleştirilmiş polinom özdeşliklerini sağlayan merkezi kapalı asal halkanın güçlü ilkel (primitive) halka olduğunu göstermiştir. $\mathrm{Bu}$ önemli sonucu ispatlamak için, Martindale, kendi adıyla anılan (sağ, sol) kesirler halkası kavramını tanımlamıştır. Daha sonra Amitsur [1] bu kavramı yarıasal halkalara

\footnotetext{
*Ahmet GENÇ, agenc@adu.edu.tr, http://orcid.org/0000-0002-2535-1689
} 
geniş̧letmiştir.

Asal gamma halkalarında klasik asal halkalar teorisinde olduğu gibi üzerinde çalıştığımız halkadan daha geniş olan ve halkanın kendisini homomorfik olarak içeren bir kesirler halkasını idealler üzerinde inşa etmektir. Bu çalışmada kesirler halkasını soldan kapalı idealler üzerinde oluşturulmuştur. İdealler üzerinden elde edilen kesirler halkasının soldan kapalı idealleri üzerinden elde edilen kesirler halkasını içerdiğini ancak gamma halka birimli ise bu iki kesirler halkasının çakıştığını ifade ettik. Kesirler halkasını, tek yanlı kapalı idealleri üzerinden oluşturmamızın sebebi, gamma halkanın tek yanlı kapalı idealleri ve operatör halkanın tek yanlı kapalı idealleri arasında birebir eşleme olması ve bunun gamma halkalarının kesirler halkası ile operatör halkasının kesirler halkası arasındaki ilişkiyi araştırabilmeyi olanak sağlamasıdır.

\section{2. Önbilgiler}

2.1 Tanım: $M$ elemanları $a, b, c, \ldots$ ile gösterilen toplamsal değişmeli grup ve $\Gamma$ elemanları $\alpha, \beta, \gamma, \ldots$ ile gösterilen toplamsal değişmeli grup olsun. Her $a, b \in M$ ve $\gamma$, $\beta \in \Gamma$ için, $a \gamma b$ M toplamsal grubunun elemanı ve $\gamma a \beta$ elemanı da $\Gamma$ toplamsal grubunun elemanı olarak tanımlansın. Eğer bu çarpımlar aşağıdaki üç koşulu da sağlanıyor ise $\mathrm{M}$ toplamsal grubuna $\Gamma$-halkası denir.

(i) $\left(a_{1}+a_{2}\right) \gamma b=a_{1} \gamma b+a_{2} \gamma b$

$a\left(\gamma_{1}+\gamma_{2}\right) b=a \gamma_{1} b+a \gamma_{2} b$

$a \gamma\left(b_{1}+b_{2}\right)=a \gamma b_{1}+a \gamma b_{2}$

(ii) $(a \gamma b) \beta c=a \gamma(b \beta c)=a(\gamma b \beta) c$

(iii) Her $a, b \in M$ için $a \gamma b=0$ ise $\gamma=0$.

Barnes [2] makalesinde Nobusawa'nın vermiş olduğu $\Gamma$-halkası tanımının koşullarını zayıflatarak aşağıdaki tanımı vermiştir.

Tanım 2.2 $M=\{a, b, c, \ldots$.$\} ve \Gamma=\{\alpha, \beta, \gamma, \ldots$.$\} toplamsal değişmeli gruplar olmak üzere,$ her $a, b, c \in M, \alpha, \beta \in \Gamma$ için,

(i) $a \alpha b, M$ grubunun elemanıdır.

(ii) $(a+b) \alpha c=a \alpha c+b \alpha c$

$a(\alpha+\beta) b=a \alpha b+a \beta b$

$a \alpha(b+c)=a \alpha b+a \alpha c$

(iii) $(a \alpha b) \beta c=a \alpha(b \beta c)$

koşullarını sağlanıyorsa $M$ toplamsal grubuna $\Gamma$-halkası denir.

Bundan sonra tanımlarda karışıklığa yol açmamak için Barnes anlamında $M \Gamma$-halkası için $(\Gamma, M)_{\mathrm{B}}$ ve Nobusawa anlamında $M \Gamma$-halkası için $(\Gamma, M)_{\mathrm{N}}$ gösterimini kullanılacaktır.

Tanım 2.3 $I \subseteq M$ olmak üzere, ( $\Gamma, \mathrm{M})$ gamma halkasının, $I \Gamma M=\{a \alpha c \mid a \in I, \alpha \in \Gamma, c \in M\}$ $(M \Gamma I)$ kümesini kapsayan $I$ toplamsal grubuna $(\Gamma, M)$ gamma halkasının săg (sol) ideali denir. Eğer $I$ hem sağ hem de sol ideal ise $I$ toplamsal grubuna $(\Gamma, M)$ gamma halkasının iki yanlı ideali veya kısaca $(\Gamma, M)$ gamma halkasının ideali denir. 
Tanım $2.4(\Gamma, M)$ в olmak üzere, $x \in M$ ve $\delta \in \Gamma$ olsun. Her $y \in M$ için, $y[\delta, x]=y \delta x$ ile tanımlanan $[\delta, x]: M \rightarrow M$ dönüşümü, $M$ toplamsal grubunun bir endomorfizmasıdır. $\delta_{i} \in \Gamma, x_{i} \in M$ olmak üzere, $\sum_{i}\left[\delta_{i}, x_{i}\right]$ formundaki tüm toplamları içeren $R$ kümesi, $\operatorname{End}^{r}(M)$ (sağ taraftan $M$ ile işleme konulan endomorfizmlerin halkası) nin bir alt halkasıdır. $R$ уе $(\Gamma, M)$ в nın săg operatör halkası denir.

Benzer şekilde, $(\Gamma, M)$ в gamma halkasının sol operatör halkası $L=\left\{\sum_{j}\left[y_{j}, \mu_{j}\right] \mid y_{j} \in M, \mu_{j} \in \Gamma\right\} \leq \operatorname{End}^{l}(M)$ tanımlanır.

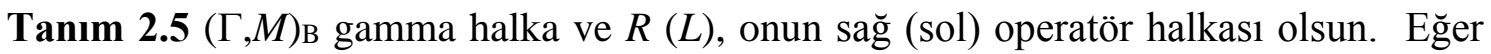
her $\quad x \in M$ için, $\sum_{i} x \delta_{i} e_{i}=x \quad\left(\sum_{j} f_{j} w_{j} x=x\right) \quad$ olacak ş̧ekilde $\quad \sum_{i}\left[\delta_{i}, e_{i}\right] \in R$ $\left(\sum_{j}\left[f_{j}, w_{j}\right] \in L\right)$ var ise $\sum_{i}\left[\delta_{i}, e_{i}\right]\left(\sum_{j}\left[f_{j}, w_{j}\right]\right)$ elemanına $M$ gamma halkasının să (sol) birimi denir. $\sum_{i}\left[\delta_{i}, e_{i}\right]$ elemanın $R$ nin birimi, $\sum_{j}\left[f_{j}, w_{j}\right]$ elemanın $L$ nin birimi olduğu kolaylıkla görülebilir.

Aynı zamanda, $\sum_{i}\left[\delta_{i}, e_{i}\right]\left(\sum_{j}\left[f_{j}, w_{j}\right]\right)$ elemanı $\Gamma M$-halkasının sol (să̆) birimidir.

Tanım 2.6 $(\Gamma, \mathrm{M})_{\mathrm{N}}$ gamma halka olsun. Her $x \in M$ için, $e \delta x=x$ olacak şekilde $e \in M$ ve $\delta \in \Gamma$ var ise $(\delta, e)$ çiftine $(\Gamma, \mathrm{M})$ gamma halkasının güçlü (strong) sol birimi denir. Her $x \in M$ için, $x \delta e=x$ olacak şekilde $e \in M$ ve $\delta \in \Gamma$ var ise $(\delta, e)$ çiftine $(\Gamma, M)$ gamma halkasının güçlü (strong) să̆ birimi denir. $(\delta, e)$ çifti, $(\Gamma$, $M)$ gamma halkasının hem güçlü sağ ve sol birim ise kısaca güçlü (strong) birim denir.

Tanım 2.7 [5] $\left(\Gamma_{1}, M_{1}\right)_{\mathrm{N}}$ ve $\left(\Gamma_{2}, M_{2}\right)_{\mathrm{N}}$ gamma halkaları, $\phi$ ve $\theta$ sirasiyla $\Gamma_{1}$ grubundan $\Gamma_{2}$ grubuna ve $\mathrm{M}_{1}$ grubundan $\mathrm{M}_{2}$ grubuna iki dönüşüm olmak üzere, aşağıdaki koşulları sağlayan $(\phi, \theta)$ siralı çiftine $\left(\Gamma_{1}, M_{1}\right)_{\mathrm{N}}$ gamma halkasından $\left(\Gamma_{2}, M_{2}\right)_{\mathrm{N}}$ gamma halkasına homomorfizma denir.

(i) $\theta, M_{1}$ grubundan $M_{2}$ grubuna grup homomorfizmasidir.

(ii) $\phi, \Gamma_{1}$ grubundan $\Gamma_{2}$ grubuna grup homomorfizmasıdır.

(iii) Her $x, y \in M$ ve $\alpha \in \Gamma$ olmak üzere,

$$
(x \alpha y) \theta=(x \theta)(\alpha \phi)(y \theta)
$$

(iv) Her $x \in M$ ve $\alpha, \gamma \in \Gamma$ olmak üzere,

$$
(\alpha x \gamma) \phi=(\alpha \phi)(x \theta)(\gamma \phi)
$$

İlaveten, $\phi$ ve $\theta$ bire bir ve örten ise $(\phi, \theta)$ sıralı çifti $\left(\Gamma_{1}, M_{1}\right)_{\mathrm{N}}$ gamma halkasından $\left(\Gamma_{2}, M_{2}\right)_{\mathrm{N}}$ üzerine izomormorfizma denir ve $\left(\Gamma_{1}, M_{1}\right)_{\mathrm{N}} \cong\left(\Gamma_{2}, M_{2}\right)_{\mathrm{N}}$ ile gösterilir. 
Tanım 2.8 Her $n, n_{1}, n_{2} \in N, m, m_{1}, m_{2} \in M$ ve $\alpha, \beta \in \Gamma$ için, aşağıdaki koşulları sağlayan $N$ toplamsal değişmeli grubuna sağ $\Gamma M-$ modül denir.

(i) $n \alpha m \in N$

(ii) $\left(n_{1}+n_{2}\right) \alpha m=n_{1} \alpha m+n_{2} \alpha m$

(iii) $n(\alpha+\beta) m=n \alpha m+n \beta m$

(iv) $n \alpha\left(m_{1}+m_{2}\right)=n \alpha m_{1}+n \alpha m_{2}$

Tanım 2.9 $N_{1}$ ve $N_{2}$ sağ $\Gamma M-$ modül olsun. Aşağıdaki koşulları sağlayan $\theta: N_{1} \rightarrow N_{2}$ dönüşümüne sağ $\boldsymbol{\Gamma} \boldsymbol{M}$ - modül homorfizması denir.

(i) $\theta: N_{1} \rightarrow N_{2}$ grup homomorfizmasidır,

(ii) Her $x \in N_{1}$ ve $m \in M, \alpha \in \Gamma$ için, $\theta(x \alpha m)=\theta(x) \alpha m$.

Tanım 2.10 $(\Gamma, M)$ gamma halka olsun. Eğer $(\Gamma, M)$ gamma halkasının herhangi iki $A$ ve $B$ ideali için, $A \Gamma B \subseteq P$ olması $A \subseteq P$ veya $B \subseteq P$ olmasını gerektiriyorsa, $(\Gamma, M)$ nın $P$ idealine asal ideal denir.

Tanım 2.11 $(\Gamma, M)$ gamma halkasının sifir ideali asal ideal ise $(\Gamma, M)$ gamma halkasına asal gamma halka denir.

Tanım 2.12 $R$ ve $L$ sirasıyla $(\Gamma, M)$ gamma halkasının sağ ve sol operatör halkaları $A, R$ halkasının sol ideali olsun. $R A=A$ ise $A$ sol idealine soldan kapalı ideal denir. $U$, $(\Gamma, M)$ gamma halkasının sol ideali olsun. $L U=M \Gamma U=U$ ise $U$ sol idealine, $M$ nin soldan kapalı ideali denir.

Benzer şekilde, $B, L$ halkasının sağ ideali olsun. $B L=B$ ise $B$ sağ idealine sağdan kapalı ideal denir. $V,(\Gamma, M)$ gamma halkasının sağ ideali olsun. $V R=V \Gamma M=V$ ise $V$ sağ idealine, $M$ nin sağdan kapalı ideali denir.

Tanım 2.13 $A, R$ sol operatör halkasının sol ideali ise $M A$ da $(\Gamma, M)$ gamma halkasının sol idealidir. $M A$ sol ideali $* A$ ile gösterilir. $U,(\Gamma, M)$ gamma halkasının sol ideali ise $[\Gamma, U]$ da $R$ sol operatör halkasının sol idealidir. $[\Gamma, U]$ sol ideali $* U$ ile gösterilir.

Benzer şekilde, $B L$ sağ operatör halkasının sağ ideali ise $B M$ de $(\Gamma, M)$ gamma halkasının sağ idealidir. $B M$ sağ ideali $B *$ ile gösterilir. $V,(\Gamma, M)$ gamma halkasının sağ ideali ise $[V, \Gamma]$ da $L$ sağ operatör halkasının sağ idealidir.[V, $\Gamma]$ sağ ideali $V *$ ile gösterilir.

Özellik 2.14 Eğer $R$ sol operatör halkasının $A$ sol ideali soldan kapalı ise $(\Gamma, M)$ gamma halkasının $* A$ sol ideali de soldan kapalıdır. Eğer $(\Gamma, M)$ gamma halkasının $U$ sol ideali soldan kapalı ise $R$ sol operatör halkasının $* U$ sol ideali de soldan kapalıdır. Benzer özellikler sağdan kapalılar içinde verilebilir[5].

Teorem 2.15 $R$ sol operatör halkasının soldan kapalı sol idealleri ile $(\Gamma, M)$ gamma halkasının soldan kapalı sol idealleri arasında birebir eşleme vardır [5]. 


\section{Asal gamma halkanın soldan kapalı idealleri ile kurulan kesirler halkası}

Örnek 3.1 $M=\left\{\left[\begin{array}{ll}a & 0 \\ a & 0\end{array}\right]: a \in \mathbb{Z}\right\}$ ve $\Gamma=\left\{\left[\begin{array}{ll}x & 0 \\ 0 & 0\end{array}\right]: x \in \mathbb{Z}\right\}$ güçlü să̆ birimli gamma halkadır. $\quad\left(\left[\begin{array}{ll}1 & 0 \\ 0 & 0\end{array}\right],\left[\begin{array}{ll}1 & 0 \\ 1 & 0\end{array}\right]\right) \in(\Gamma, M)_{N}$ gamma halkasının güçlü să birimidir. $U=\left\{\left[\begin{array}{ll}3 a & 0 \\ 3 a & 0\end{array}\right]: a \in \mathbb{Z}\right\}(\Gamma, M)_{N}$ gamma halkasının soldan kapalı bir idealidir.

$(\Gamma, M)_{\mathrm{N}}$, güçlü sağ birimli asal gamma halka ve $U,(\Gamma, M)_{\mathrm{N}}$ gamma halkasının sıfırdan farklı soldan kapalı bir ideali olsun. $(\Gamma, M)_{\mathrm{N}}$, sağ güçlü birimli olduğundan $M \Gamma M=M$ ve Tanım 2.6 dan $R$ operatör halkası birimlidir. $M \Gamma M=M$ olması kullanılarak, $R^{2}=[\Gamma, M] .[\Gamma, M]=[\Gamma, M \Gamma M]=[\Gamma, M]=R$ elde edilir. Yani $R$ operatör halkası da kendisinin soldan kapalı bir idealidir. $A$ ve $B, R$ operatör halkasının soldan kapalı idealleri olmak üzere, $R(A \cap B) \subseteq A \cap B$ olduğu $A \cap B$ nin ideal olmasından açıktır. $A \cap B \subseteq R(A \cap B)$ olduğu $R$ operatör halkasının birimli olmasından görülür. O halde, soldan kapalı ideallerin arakesiti de soldan kapalı bir idealdir. Özellik 2.14 ve Teorem 2.15 kullanılarak, $(\Gamma, M)_{\mathrm{N}}$ gamma halkasının soldan kapalı ideallerinin arakesitinin de soldan kapalı bir ideal olduğu kolaylıkla görülür.

Özellik 3.2 $(\Gamma, M)_{\mathrm{N}}$ gamma halkasının sıfirdan farklı soldan kapalı $U$ ve $V$ idealleri için, $U \Gamma V$ ideali de soldan kapalıdır ve $U \Gamma V \subseteq U \cap V$ dir.

İspat $U$ soldan kapalı olduğundan $M \Gamma U \Gamma V=U \Gamma V$ ve $\mathrm{U}$ ve $\mathrm{V}$ ideal olması kullanılarak $U \Gamma V \subseteq U$ ve $U \Gamma V \subseteq V$ elde edilir. Yani, $U \Gamma V \subseteq U \cap V$ dir.

$U,(\Gamma, M)_{\mathrm{N}}$ gamma halkasının sıfirdan farklı soldan kapalı bir ideali olsun.

$$
\aleph=\{(U, f) \mid f: U \rightarrow M f, \text { sol } M \Gamma-\text { modül homomorfizma }\}
$$

kümesi üzerinde, “ ” bağıntısı “ $\left.(U, f) \sim(V, g) \Leftrightarrow f\right|_{W}=\left.g\right|_{W}$ olacak şekilde $(\Gamma, M)_{\mathrm{N}}$ gamma halkasının $U \cap V$ tarafindan kapsanan sıfırdan farklı en az bir soldan kapalı $W$ ideali vardır" olarak tanımlansın. $(\Gamma, M)_{\mathrm{N}}$ asal gamma halka olduğundan, sıfırdan farklı soldan kapalı $W$ ideali bulmak her zaman mümkündür. Örneğin, eğer $W=U \Gamma V$ alırsak, $U \neq 0, V \neq 0$ ve $U, V(\Gamma, M)_{\mathrm{N}}$ gamma halkasının ideali ve $(\Gamma, M)_{\mathrm{N}}$ asal gamma olduğundan $0 \neq U \Gamma V$ olur. Özellik 3.2 den $U \Gamma V \subseteq U \cap V$ ve $U \Gamma V,(\Gamma, M)_{\mathrm{N}}$ gamma halkasının soldan kapalı bir idealdir.

a) $0 \neq U(\Gamma, M)_{\mathrm{N}}$ gamma halkasının ideali için, $W=U \Gamma U$ alındığında $W \neq 0$ dır. $(U, f) \sim(U, f)$ olduğu açıktır. Yani “ ” bağıntısı yansıma özelliğini sağlar.

b) $(U, f) \sim(V, g)$ olsun. $\left.f\right|_{W}=\left.g\right|_{W}$ olacak şekilde $(\Gamma, M)_{\mathrm{N}}$ gamma halkasının $U \cap V$ tarafından kapsanan sıfırdan farklı en az bir $W$ ideali vardır. Buradan, $(V, g) \sim$ $(U, f)$ elde edilir. Bu da “ " bağıntısının simetrik olduğunu gösterir.

c) $(U, f) \sim(V, g)$ ve $(V, g) \sim(K, h)$ olsun. $(U, f) \sim(V, g)$ olduğundan $(\Gamma, M)_{\mathrm{N}}$ gamma halkasının sıfırdan farklı bir $W_{1} \subseteq U \cap V$ ideali için, $\left.f\right|_{W_{1}}=\left.g\right|_{W_{1}}$ eşitliği sağlanır. $(V, g) \sim(K, h)$ olduğundan $(\Gamma, M)_{\mathrm{N}}$ gamma halkasının sıfırdan farklı bir $W_{2} \subseteq V \cap K$ ideali için, $\left.g\right|_{W_{2}}=\left.h\right|_{W_{2}}$ olur. $W=W_{1} \Gamma W_{2}$ alalım. $W,(\Gamma, M)_{\mathrm{N}}$ 
gamma halkasının sıfırdan farklı soldan kapalı idealidir ve $W=W_{1} \Gamma W_{2}$ $\subseteq W_{1} \cap W_{2} \subseteq U \cap V \cap K \subseteq U \cap K$ sağlanır. Diğer taraftan, $\left.f\right|_{W_{1}}=\left.g\right|_{W_{1}}$ ve $\left.g\right|_{W_{2}}=\left.h\right|_{W_{2}}$ olduğundan $\left.f\right|_{W}=\left.g\right|_{W}$ ve $\left.g\right|_{W}=\left.h\right|_{W}$ olduğu açıktır. O halde, $(\Gamma$, $M)_{\mathrm{N}}$ gamma halkasının sıfırdan farklı $W=W_{1} \Gamma W_{2}$ soldan kapalı ideali için $\left.f\right|_{W}=\left.h\right|_{W}$ dır. Böylece, $(U, f) \sim(K, h)$ olduğu görülür. Yani “ ” bağıntısı geçişmelidir.

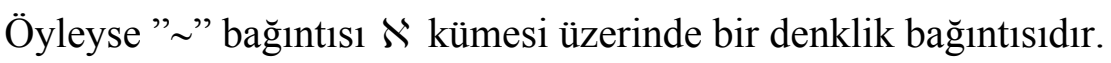

Denklik sınıflarını $\overline{(U, f)}=\hat{f}$ ile gösterelim.

$\aleph$ kümesinin tüm elemanlarının denklik sınıflarının kümesi $Q_{C l}$ olsun.

$Q_{C l}$ üzerinde "+" işlemi aşağıdaki gibi tanımlansın.

$\hat{f}+\hat{g}=\overline{(U, f)}+\overline{(V, g)}=\overline{(U \cap V, f+g)}$

Yukarıda tanımlanan toplama işlemi iyi tanımlıdır. $\overline{(U, f)}, \overline{(V, g)}, \overline{\left(U^{\prime}, f^{\prime}\right)}$, $\overline{\left(V^{\prime}, g^{\prime}\right)} \in Q$ için, $\quad(\overline{(U, f)}, \overline{(V, g)})=\left(\overline{\left(U^{\prime}, f^{\prime}\right)}, \overline{\left(V^{\prime}, g^{\prime}\right)}\right) \quad$ olsun. $\quad$ O halde, $\overline{(U, f)}=\overline{\left(U^{\prime}, f^{\prime}\right)}$ ve $\overline{(V, g)}=\overline{\left(V^{\prime}, g^{\prime}\right)} \quad$ dir. $\quad$ Buradan, $(U, f) \sim\left(U^{\prime}, f^{\prime}\right) \quad$ ve $(V, g) \sim\left(V^{\prime}, g^{\prime}\right)$ elde edilir. “ $\sim$ " bağıntısının tanımından, $W_{1} \subseteq U \cap U^{\prime}$ olacak şekilde $(\Gamma, M)_{\mathrm{N}}$ gamma halkasının sifirdan farklı soldan kapalı bir $W_{1}$ ideali üzerinde $f=f^{\prime}$ ve $W_{2} \subseteq V \cap V^{\prime}$ olacak şekilde $(\Gamma, M)_{\mathrm{N}}$ gamma halkasının sıfırdan farklı soldan kapalı bir $W_{2}$ ideali üzerinde $g=g^{\prime}$ dir. $W=W_{1} \Gamma W_{2}$ alalım. $W \neq 0$ ve $W \subseteq(U \cap V) \cap\left(U^{\prime} \cap V^{\prime}\right)$ dır. Her $w \in W$ için, $(w)(f+g)=(w) f+(w) g=(w) f^{\prime}+(w) g^{\prime}=(w)\left(f^{\prime}+g^{\prime}\right)$ olduğundan, $(U \cap V, f+g) \sim\left(U^{\prime} \cap V^{\prime}, f^{\prime}+g^{\prime}\right)$ bulunur. O halde, $\overline{(U \cap V, f+g)}=\overline{(\mathrm{W} \cap T, h+k)}$ elde edilir.

Burada $f+g: U \cap V \rightarrow M$ sol $M \Gamma$-modül homomorfizmasıdır. $Q_{C l}$ kümesinin bu işlem altında toplamsal değişmeli grup olduğu bir önceki bölüme benzer şekilde kolaylıkla gösterilir.

Benzer şekilde, $(M, \Gamma)_{\mathrm{N}}$ asal gamma halka ve $0 \neq \Omega(M, \Gamma)_{\mathrm{N}}$ gamma halkasının soldan kapalı bir ideali olsun.

$\mathfrak{R}=\{(\Omega ; \tau) \mid \tau: \Omega \rightarrow \Gamma \tau$, sağ $M \Gamma$ - modül homomorfizması $\} \mathfrak{R}$ kümesi üzerinde “ $\approx$ " bağıntısı şu şekilde tanımlansın.

“( $\left.(\Omega, \tau) \approx(\Lambda, \sigma) \Leftrightarrow \tau\right|_{\Pi}=\left.\sigma\right|_{\Pi}$ olacak şekilde $(M, \Gamma)_{\mathrm{N}}$ gamma halkasının $\Omega \cap \Lambda$ tarafindan kapsanan sıfırdan farklı en az bir soldan kapalı $\Pi$ ideali vardır”

$(M, \Gamma)_{\mathrm{N}}$ asal gamma halka olduğundan, sifirdan farklı $(M, \Gamma)_{\mathrm{N}}$ gamma halkasının $\Pi$ ideali her zaman bulunabilir. Yukarıdaki bağıntıya benzer şekilde " $\approx$ " bağıntısının denklik bağıntısı olduğu görülür. 
Denklik sınıflarını $\overline{(\Omega, \tau)}=\hat{\tau}$ ile gösterelim. $\mathfrak{R}$ kümesinin bütün elemanlarının denklik sınıflarının kümesini $\Delta_{C l}$ ile gösterelim. $\Delta_{C l}$ üzerinde toplama işlemini aşağıdaki gibi tanımlayalım.

$$
\hat{\tau}+\hat{\sigma}=\overline{(\Omega, \tau)}+\overline{(\Lambda, \sigma)}=\overline{(\Omega \cap \Lambda, \tau+\sigma)}
$$

Yukarıda yaptıklarımıza benzer şekilde $\Delta_{C l}$ kümesinin toplamsal değişmeli grup olduğu kolaylıkla görülür.

$\tau, \Omega$ dan $\Gamma$ ya sağ $M \Gamma-$ modül homomorfizması olmak üzere, $\tau$ yardımı ile toplamsallık özelliğini sağlayacak şekilde $\stackrel{\Delta}{\tau}: M \Omega M \rightarrow M, \stackrel{\Delta}{\tau}(\operatorname{m} \gamma \mathrm{n})=\operatorname{m} \tau(\gamma) \mathrm{n}$ ile $\stackrel{\Delta}{\tau}$ dönüşümünü tanımlayalım.

$Q_{C l}$ ve $\Delta_{C l}$ toplamsal değişmeli grupları göz önüne alınarak aşağıdaki işlemini tanımlayalım;

.$: Q_{C l} \times \Delta_{C l} \times Q_{C l} \rightarrow Q_{C l}$ işlemini,

$(\hat{f}, \hat{\tau}, \hat{g}) \rightarrow \hat{f} \cdot \hat{\tau} \cdot \hat{g}=\overline{(U, f)} \overline{(\Omega, \tau)} \overline{(V, g)}=\overline{(V \Omega U, f \stackrel{\Delta}{\tau} g)}$ ile tanımlayalım.

$\mathrm{Bu}$ işlemin iyi tanımlı olduğunu gösterelim.

$(\overline{(U, f)}, \overline{(\Omega, \tau)}, \overline{(V, g)})=\left(\overline{\left(U_{1}, f_{1}\right)}, \overline{\left(\Omega_{1}, \tau_{1}\right)}, \overline{\left(V_{1}, g_{1}\right)}\right)$ olsun.

“ " ve “ $\approx$ ” bağıntısının tanımından,

$\left.(U, f) \sim\left(U_{1}, f_{1}\right) \Rightarrow f\right|_{W}=\left.f_{1}\right|_{W}$ olacak şekilde, $(\Gamma, M)_{\mathrm{N}}$ gamma halkasının $U \cap U_{1}$ tarafından kapsanan sıfirdan farklı en az bir soldan kapalı $W$ ideali vardır, $\left.(\Omega, \tau) \approx\left(\Omega_{1}, \tau_{1}\right) \Rightarrow \tau\right|_{\Pi}=\left.\tau_{1}\right|_{\Pi}$ olacak şekilde, $(M, \Gamma)_{\mathrm{N}}$ gamma halkasının $\Omega \cap \Omega_{1}$ tarafından kapsanan sifirdan farklı en az bir soldan kapalı $\Pi$ ideali vardır ve $\left.(V, g) \sim\left(V_{1}, g_{1}\right) \Rightarrow g\right|_{K}=\left.g_{1}\right|_{K}$ olacak şekilde, $(\Gamma, M)_{\mathrm{N}}$ gamma halkasının $V \cap V_{1}$ tarafindan kapsanan sıfirdan farklı en az bir soldan kapalı $K$ ideali var olduğu elde edilir. $A=K \Pi W \Gamma K \Pi W \neq 0$. Eğer $A=0$ olsa $(\Gamma, M)_{\mathrm{N}}$ asal olması ve $K \Pi W$ in $(\Gamma, \mathrm{M})_{\mathrm{N}}$ halkasının ideali olması kullanılarak $\Pi=0$ elde edilir. Bu da $\Pi \neq 0$ olması ile çelişir.

$$
\begin{aligned}
A=K \Pi W \Gamma K \Pi W= & \left\{\sum_{\text {sonlu }} k \gamma w \beta k^{\prime} \gamma^{\prime} w^{\prime} \mid \forall w, w^{\prime} \in W, \gamma, \gamma^{\prime} \in \Pi, k, k^{\prime} \in K, \beta \in \Gamma\right\} . \\
\left(k \gamma w \beta k^{\prime} \gamma^{\prime} w^{\prime}\right) f \stackrel{\Delta}{\tau} g & =\left(k \gamma w \beta k^{\prime} \gamma^{\prime} f\left(w^{\prime}\right)\right) \stackrel{\Delta}{\tau} g=\left(k \tau\left(\gamma w \beta k^{\prime} \gamma^{\prime}\right) f\left(w^{\prime}\right)\right) g \\
& =\left(k \tau_{1}\left(\gamma w \beta k^{\prime} \gamma^{\prime}\right) f_{1}\left(w^{\prime}\right)\right) g_{1} \\
& =\left(k \gamma w \beta k^{\prime} \gamma^{\prime} w^{\prime}\right) f_{1} \stackrel{\Delta}{\tau}_{1} g_{1}, \forall w, w^{\prime} \in \mathrm{W}, \gamma, \gamma^{\prime} \in \Pi, k, k^{\prime} \in K, \beta \in \Gamma .
\end{aligned}
$$

Ayrıca, $f_{1} \stackrel{\Delta}{\tau_{1}} g_{1}$ toplamsal olduğundan, $\left.f \stackrel{\Delta}{\tau} g\right|_{A}=\left.f_{1} \dot{\tau}_{1} g_{1}\right|_{A}$ olduğu görülür.

$\left(\Delta_{C l}, Q_{C l}\right)_{\mathrm{N}}$ nun gamma halka olduğunu Genç [4] benzer olarak gösterilir. Böylece, $\left(\Delta_{C l}\right.$, $\left.Q_{C l}\right)_{\mathrm{N}}$ gamma halkadır. Benzer şekilde aşağıdaki işlem altında $\left(Q_{C l}, \Delta_{C l}\right)_{\mathrm{N}}$ nın gamma halka olduğu görülebilir. 
$(\hat{\tau}, \hat{f}, \hat{\sigma}) \rightarrow \hat{\tau} \cdot \hat{f} \cdot \hat{\sigma}=\overline{(\Omega, \tau)} \overline{(U, f)} \cdot \overline{(\Lambda, \sigma)}=\overline{(\Lambda U \Omega, \tau \hat{f} \sigma)}$ olacak şekilde

.$: \Delta_{C l} \times Q_{C l} \times \Delta_{C l} \rightarrow \Delta_{C l}$ “.” işlemi ve burada $\stackrel{\Delta}{f}: \Gamma U \Gamma \rightarrow \Gamma(\gamma m \beta) \stackrel{\Delta}{f}=\gamma f(m) \beta$ olarak tanımlanır.

$\left(\Delta_{C l}, Q_{C l}\right)_{\mathrm{N}}$ gamma halkası birimlidir. $I_{M}: M \rightarrow M$ ve $I_{\Gamma}: \Gamma \rightarrow \Gamma$ birim dönüşümleri $M \Gamma$-modül homomorfimasıdır. Her $\overline{(U, f)} \in Q_{C l}$ için, $\overline{(U, f)} \cdot \overline{\left(\Gamma, I_{\Gamma}\right)} \cdot \overline{\left(M, I_{M}\right)}=\overline{\left(M, I_{M}\right)} \cdot \overline{\left(\Gamma, I_{\Gamma}\right)} \cdot \overline{(U, f)}=\overline{(U, f)}$ $\left(\overline{\left(M, I_{M}\right)}, \overline{\left(\Gamma, I_{\Gamma}\right)}\right)$ çifti $\left(\Delta_{C l}, Q_{C l}\right)_{\mathrm{N}}$ halkasının birimidir.

Sonuç 3.3 $\left(\Delta_{C l}, Q_{C l}\right)_{\mathrm{N}}$ kesirler halkası $(\Delta, Q)_{\mathrm{N}}$ kesirler halkası tarafından kapsanır. Eğer $(\Gamma, M)_{\mathrm{N}}$ asal gamma halkası güçlü birimli ise bu halkalar birbirine eşittir.

İspat: $\left(\Delta_{C l}, Q_{C l}\right)_{\mathrm{N}}$ kesirler halkası kapalı idealler üzerinden kurulduğundan ve her kapalı ideal öncelikle ideal olarak alındığından $\left(\Delta c l, Q_{C l}\right)_{\mathrm{N}} \subset(\Delta, Q)_{\mathrm{N}}$ olduğu açıktır. Ĕger $(\Gamma, M)_{\mathrm{N}}$ asal gamma halkası güçlü birimli ise her ideali kapalı olduğundan $\left(\Delta C l, Q_{C l}\right)_{\mathrm{N}}$ $=(\Delta, Q)_{\mathrm{N}}$ olduğu kolaylikla görülür.

\section{Sonuç}

$\mathrm{Bu}$ çalışmada, asal gamma halkalarının soldan kapalı idealler üzerinde kesirler halkası oluşturulmuş ve bu halkanın birimli olduğu, asal gamma halkanın kesirler halkası tarafından kapsandığı ve eğer asal gamma halkası güçlü birimli ise bu iki kesirler halkasının eşit olduğu gösterilmiştir.

\section{Kaynaklar}

[1] Amitsur, S. A., On central division algebras, Israel J. Math., 12, 408-420, (1972).

[2] Barnes, W. E., On the $\Gamma$-ring of Nobusawa, Pacific Journal of Mathematics. 18, 411-422, (1966).

[3] Beidar K. I., Martindale III W. S. and Mikhalev, A. V., Rings with Generalized Identities, Marcel Dekker Incoporated, (1996).

[4] Genç A., Asal Gamma halkalarının Kesirler Halkası, Doktora Tezi, Ege Üniversitesi, Fen Bilimleri Enstitüsü, İzmir, (2008).

[5] Kyuno, S., Gamma Rings, Hadronic Press Incorporated, (1991).

[6] Martindale, W., Prime Rings Satisfying a Generalized Polynomial Identity, Journal of Algebra, 12, 576-584, (1969).

[7] Nobusawa, N., On a Generalization of the Theory, Osaka Journal of Mathematics, 1, 81-89, (1964). 